\title{
SiO emission from a huge, detached shell in IRC $+10420^{\star}$
}

\author{
A. Castro-Carrizo ${ }^{1}$, R. Lucas ${ }^{2}$, V. Bujarrabal ${ }^{1}$, F. Colomer ${ }^{1}$, and J. Alcolea ${ }^{1}$ \\ 1 Observatorio Astronómico Nacional (IGN), Apdo. 1143, 28800 Alcalá de Henares, Spain \\ e-mail: (bujarrabal, colomer, j.alcolea) @oan.es \\ 2 IRAM, 300 rue de la Piscine, 38406 St Martin d'Hères, France \\ e-mail: lucas@iram.fr
}

Received 28 November 2000 / Accepted 6 February 2001

\begin{abstract}
We present observations of the $v=0 J=2-1$ thermal emission of $\mathrm{SiO}$ from the very luminous evolved star IRC +10420, performed with the Plateau de Bure interferometer. Our high-resolution maps show that the emission comes from a huge hollow shell, with a typical radius of $\sim 10^{17} \mathrm{~cm}$, that is expanding at $\sim 35 \mathrm{~km} \mathrm{~s}^{-1}$. This surprising result is strengthened by model fitting of the flux distribution, that explains the observations assuming that the width of the shell is not larger than half its typical radius. The origin of this feature is discussed.
\end{abstract}

Key words. stars: individual: IRC +10420 - (stars:) circumstellar matter - stars: AGB y post-AGB - radio lines: stars - stars: mass-loss

\section{Introduction}

\section{1. $I R C+10420$}

IRC +10420 is a very luminous star recently classified as a "yellow hypergiant" (de Jager 1998; Jones et al. 1993), with a luminosity of $\sim 710^{5} L_{\odot}$ at a distance of $\sim 5 \mathrm{kpc}$. These objects are post-red supergiants that are undergoing a fast evolution towards a higher surface temperature. The spectral type of IRC +10420 is F8Ia, although there are indications of a recent increase in temperature, up to A5 type (Klochkova et al. 1997).

IRC +10420 is surrounded by a very heavy circumstellar envelope, detected at several wavelengths. HST images of the optical light scattered by circumstellar grains (Humphreys et al. 1997) show a nebula extending almost $15^{\prime \prime}$; an inner bright region, about $3^{\prime \prime}$ wide. NIR polarimetry (Kastner \& Weintraub 1995) also shows a reflecting dust envelope extended 15"; the inner feature seen in the visible is detected in IR images between 8 and $13 \mu \mathrm{m}$ (Humphreys et al. 1997; Meixner et al. 1999). On the other hand, the inner radius of this dust shell seems to be very small, $\sim 0$ '! 13, as suggested by high resolution observations in the NIR (Ridgway et al. 1986).

Molecular observations of IRC +10420 also show an extended envelope. $\mathrm{OH}$ maser spots are distributed in a (hollow) shell with a radius of $1^{\prime \prime}-1$."5 and an expansion

Send offprint requests to: A. Castro-Carrizo,

e-mail: carrizo@oan.es

* Figure 2 is only available in electronic form at http://www.edpsciences.org velocity of about $31 \mathrm{~km} \mathrm{~s}^{-1}$ (Nedoluha \& Bowers 1992). Neri et al. (1998) detected CO line emission within a diameter of about $25^{\prime \prime}$; the total width of the $\mathrm{CO}$ profiles is $\sim 80 \mathrm{~km} \mathrm{~s}^{-1}$. From the CO data, Kastner \& Weintraub (1995) and Neri et al. (1998) deduce a very high mass loss rate, $\sim 510^{-4} M_{\odot} \mathrm{yr}^{-1}$, corresponding to an envelope with a mass of $\sim 5 M_{\odot}$ (see Sect. 3). IRC +10420 is very probably oxygen-rich, as shown by the presence of $\mathrm{OH}$ masers and the general characteristics of its molecular thermal emission.

\subsection{Thermal SiO emission}

The thermal ${ }^{28} \mathrm{Si}^{16} \mathrm{O}$ emission (from rotational transitions in the ground vibrational state) is known to show different properties than other molecular lines (Bujarrabal et al. 1989; Lucas et al. 1992). In O-rich AGB stars, SiO emission comes from the very inner regions of the envelope, within a radius $\sim 10^{15} \mathrm{~cm}$. The peculiar spectral profiles and dependence of the emitting region size on the LSR velocity are interpreted as showing that the $\mathrm{SiO}$ emission comes from a region in which the expansion velocity has not yet attained its final value. In C-rich star envelopes, the $\mathrm{SiO}$ emission is more extended and seems to come from regions presenting the final expansion velocity, up to $\sim 210^{16} \mathrm{~cm}$; this result is explained assuming that carbonaceous grains form before full silicon depletion, allowing the fast envelope expansion. Anyway, in all the objects mapped by Lucas et al. (1992), including C-rich objects, the $\mathrm{SiO}$ emission is strongly peaked in the central position and no sign of central minimum was found in the 
brightness distribution; note that chemical models do not predict an increase of the $\mathrm{SiO}$ abundance in outer shells (e.g. Willacy \& Millar 1997; MacKay \& Charnley 1999). No "detached shell" had been detected in thermal $\mathrm{SiO}$ emission, even in those objects showing detached shells in CO (Olofsson et al. 1996; Bujarrabal \& Cernicharo 1994).

\section{Observations and data reduction}

Observations of the $v=0 J=2-1$ transition of $\mathrm{SiO}$ (at a rest frequency of $86846.998 \mathrm{MHz}$ ) were carried out with the IRAM 5-antenna interferometer at Plateau de Bure (near Gap, France) towards IRC +10420 (J2000 coordinates $\left.19^{\mathrm{h}} 26^{\mathrm{m}} 48.09,+11^{\mathrm{o}} 21^{\prime} 17^{\prime \prime} 1\right)$. Five runs (snapshot) were performed using four different interferometer configurations, in February, March, and October 1999, with baselines extending up to $\sim 400 \mathrm{~m}$. The antennas were equipped with $3 \mathrm{~mm}$ cooled SIS receivers, tuned for LSB with typical SSB system temperatures of $\sim 150 \mathrm{~K}$ at the observing frequency. The PdBI correlator was configured to provide two $20 \mathrm{MHz}$ backends that were setup to slightly overlap, for a spectral resolution of $0.27 \mathrm{~km} \mathrm{~s}^{-1}$.

Phase and amplitude calibration was performed in the standard way by observing $1923+210$. The flux density scale was derived from observations of BL Lac $(2.9 \mathrm{Jy})$ and 3C 273 (19.5 Jy). The data were calibrated and analyzed with the GILDAS software package developed at IRAM and Observatoire de Grenoble. The width of the CLEANed beam (half-power contour) is $2^{\prime \prime} .5 \times 1$ ". 4 , at a position angle of $26^{\circ}$. In Fig. 1 we show the resulting maps, after compressing the velocity channels to get a velocity resolution of $4.86 \mathrm{~km} \mathrm{~s}^{-1}$.

\section{Discussion}

\subsection{The total extent and mass of the molecular envelope}

The best way to measure the total mass and mass loss rate of a circumstellar envelope is by applying the well studied $\mathrm{CO}$ excitation and chemistry models to observations of its rotational lines. We follow the method described by Loup et al. (1993), that takes into account the extent of the CO shell (limited by photodissociation), as well as opacity and excitation effects. The $\mathrm{CO} J=1-0$ data of IRC +10420 from Neri et al. (1998) were used. We adopt an expansion velocity of $35 \mathrm{~km} \mathrm{~s}^{-1}$, a distance of $5 \mathrm{kpc}$ (Sect. 1), and a $\mathrm{CO}$ abundance of $410^{-4}$, a typical value for O-rich evolved stars. Our procedure yields a mass loss rate equal to $3.610^{-4} M_{\odot} \mathrm{yr}^{-1}$ and an outer $\mathrm{CO}$ radius equal to $1.310^{18} \mathrm{~cm}$. This radius is equivalent to $17^{\prime \prime}$, only slightly larger than the outermost radius up to which $\mathrm{CO}$ has been detected. From these figures we obtain a total dynamical time for the formation of the envelope of $\sim 1.210^{4} \mathrm{yr}$ and a total mass for the molecular envelope of $4 M_{\odot}$. Our results are similar within a factor of two to those deduced by Neri et al. (1998) and Kastner \& Weintraub (1995), also from CO data. Loup et al. (1993) obtained a mass loss rate of $7.710^{-5} M_{\odot} \mathrm{yr}^{-1}$, the discrepancy with our results being mainly due to the lower distance adopted by those authors, $1.9 \mathrm{kpc}$. Our value of the mass loss rate is also very similar to that deduced from modeling of the FIR dust emission (for the same distance and expansion velocity used here) by Hrivnak et al. (1989). The inner radius of the dust shell deduced from that model is $1.2510^{16} \mathrm{~cm}$, equivalent to 0 .' 16 , comparable to that deduced from NIR observations of light scattered by dust.

\subsection{The SiO shell around IRC +10420}

Our maps of $\mathrm{SiO} v=0 J=2-1$ emission systematically show a minimum for the central velocities, that disappears at the edges of the profile, for which only a compact clump is found. If we assume that the envelope of IRC +10420 is expanding, these results can only be explained if the $\mathrm{SiO}$ emission comes from a shell, inside which weak $\mathrm{SiO}$ emission occurs. The lack of $\mathrm{SiO}$ emission from the central region of the envelope can be due to a shell-like distribution of matter or to chemical effects. It is not probable that the ring-like appearance of the $\mathrm{SiO}$ maps is due to strong differences in the line excitation in the different envelope regions, since $\mathrm{SiO}$ emission needs relatively high densities to be excited and should then come from the dense inner regions (in the absence of the two above effects); this is indeed what is observed in most stars.

For the distance adopted for IRC $+10420,5 \mathrm{kpc}$, this ring-like region has a radius of about $10^{17} \mathrm{~cm}$. Both this extremely large radius and the hollow structure are absolutely unexpected for $\mathrm{SiO}$ thermal emission from evolved star envelopes (Sect. 1.2). It is remarkable that the observed $\mathrm{SiO}$ ring is very similar in size to that found from observations of $\mathrm{OH}$ maser lines (Sect. 1.1). Note that the shell distribution of the $\mathrm{OH}$ brightness in evolved stars is mainly due to chemical effects (e.g. Goldreich \& Scoville 1976). It is also noticeable that the total $\mathrm{SiO}$ extent detected in our observations is significantly smaller than the total CO extent, about $25^{\prime \prime}$ (Sect. 1.1), and that the inner radius of the $\mathrm{SiO}$ layer is several times larger than the inner radius of the dust shell (Sect. 3.1).

The lack of knowledge on the distributions of total density and $\mathrm{SiO}$ abundance prevents sensible calculations on the $\mathrm{SiO}$ excitation. The kinetic temperature of this peculiar envelope is also unknown, since existing models for circumstellar thermodynamics only hold under quite different conditions.

Accordingly, we modeled the $\mathrm{SiO}$ emission by means of a simplified parametrization of the geometry and excitation in the emitting region. We assume that $\mathrm{SiO}$ emission comes from a shell, whose inner and outer radii are free model parameters. The excitation, described by means of a rotational temperature, is also a free parameter. The $\mathrm{SiO}$ abundance, $X(\mathrm{SiO})$, is taken to be constant in the shell; we took $X(\mathrm{SiO})=210^{-5}$, a value characteristic of the inner regions of AGB envelopes in which $\mathrm{SiO}$ is not locked in grains (Bujarrabal et al. 1989; Lucas et al. 1992). 


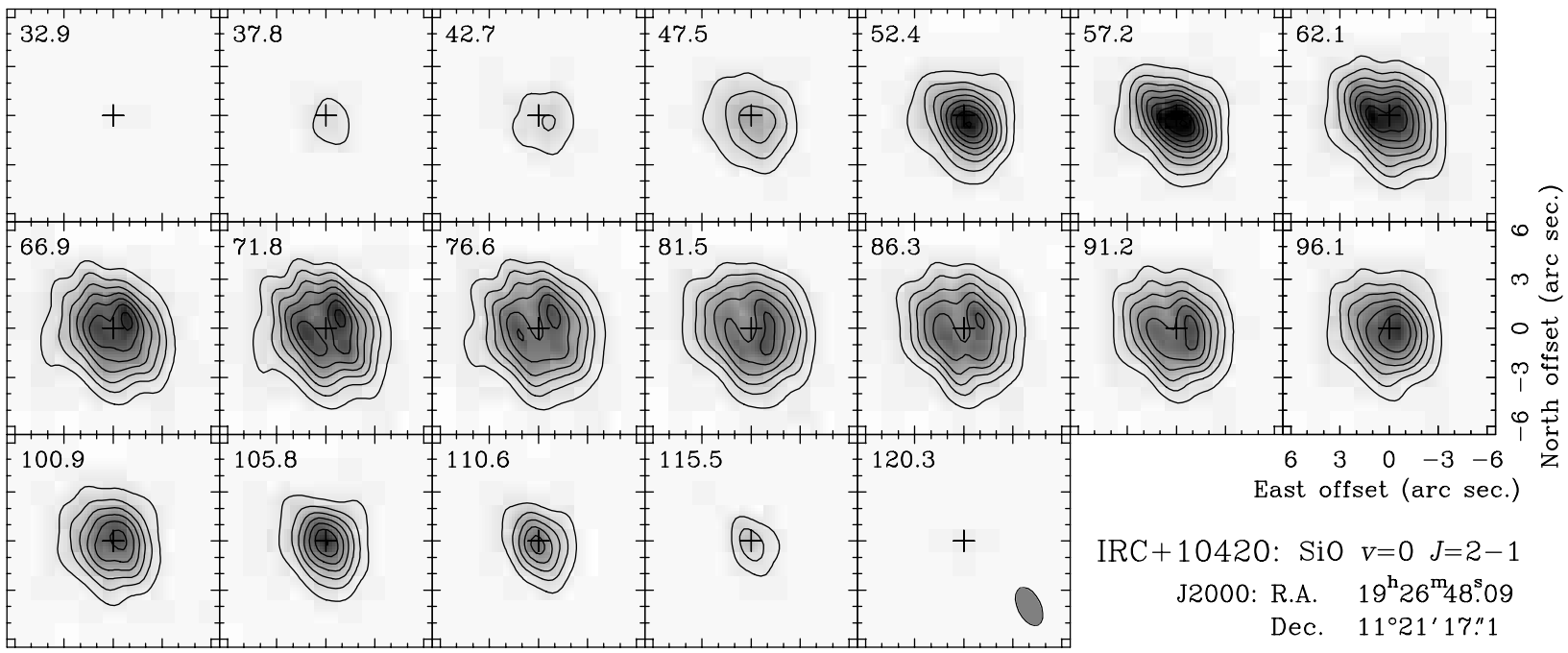

Fig. 1. PdBI maps of the $\mathrm{SiO} v=0 J=2-1$ intensity in IRC +10420 for the LSR velocities indicated in the left-upper corners. The level step is $3.7 \mathrm{~K}$ (0.08 Jy/Beam), in brightness temperature units. The CLEANed beam (half-power contour) is drawn in the right-bottom corner of the last channel plot. In Fig. 2 (in the electronic version) the results of our model fitting are shown with the same velocity channels, spatial offsets and intensity scales

A constant expansion velocity is assumed in the relevant region, as well as a local velocity dispersion of $5 \mathrm{~km} \mathrm{~s}^{-1}$. The density is calculated in order to lead to the measured mass loss rate (see Sect. 3.1); the density therefore varies (within the model shell) as the inverse square of the radius. With these ingredients, we calculate the opacity and brightness temperature for a set of observed $L S R$ velocities and impact parameters, after which we carry out the convolution with the CLEANed beam of the observations. The code calculating the synthetic maps is similar to that described in more detail in previous papers (e.g. Bujarrabal et al. 1998).

Our model readily gives results satisfactorily comparable to the observations, except for some minor features discussed below. We can reproduce our maps assuming a shell with inner and outer radii equal to $0.910^{17} \mathrm{~cm}$ and $1.410^{17} \mathrm{~cm}$, respectively. The rotational temperature given by the model fitting is of the order of $55 \mathrm{~K}$, slightly larger than the observed brightness temperature due to beam dilution. As expected, the model predictions are in clear disagreement with observations when the inner radius is smaller than $\sim 610^{16} \mathrm{~cm}$, even if we neglect the expected increase of the rotational temperature when the distance to the central star decreases. In our calculations we assumed that the density in the central regions is low; the central density must be $\lesssim 1 / 5$ of the shell average density to have a satisfactory fitting. With values of $X(\mathrm{SiO})$ larger than $\sim 10^{-6}$ the emission is optically thick, so the results depend only slightly on $X(\mathrm{SiO})$. For lower values of $X(\mathrm{SiO})$, the observations can be reproduced also, but assuming higher rotational temperatures. A temperature of $55 \mathrm{~K}$ seems to be high for the rotational excitation; the kinetic temperatures of AGB envelopes, for instance, are not expected to be so high at $10^{17} \mathrm{~cm}$. (Note that IRC +10420 is not an ordinary late-type AGB star.) The expansion velocity given by the fitting is $31 \mathrm{~km} \mathrm{~s}^{-1}$, equal to that obtained from OH data by Nedoluha \& Bowers (1992).

The maps predicted by these models are shown in Fig. 2 (in the electronic version). The twin maxima found for the central velocities, and so the apparent bipolarity in both observation and model, are due to the convolution with the observational beam. The observations show a relative increase of intensity towards the south-west, probably due to inhomogeneities in the $\mathrm{SiO}$ shell that we will not try to fit. Our model cannot fit either the observed line wings in their total spectral extent, since such spatially compact emission over wide velocity ranges should require a new ad hoc component; such wings also appeared in the $\mathrm{OH}$ maser data by Nedoluha \& Bowers (1992), mostly at red wavelengths, and could not be fitted by their simple shell model. The observed maps also show a low-intensity halo extending up to $8^{\prime \prime}$ in diameter, that would require another ad hoc component in our model. We note that uncertainties in the CLEANing also tend to introduce some spurious spatial dispersion in the maps.

In order to check that our main results are not seriously affected by the CLEANing procedure for the central velocities, we have fitted the visibility amplitude versus the $u v$ radius to the Fourier transform of different brightness distributions: Gaussian, ring and disk functions, as well as combinations of them. The contribution of a ring with an inner radius similar to that used in our model is necessary to fit the data. The best fitting is obtained when the ring is modified in such a way that the brightness in the center is about a half of the maximum. Therefore, our fitting of the visibilities confirms the presence of a central minimum in the brightness distribution for the central velocities.

In Fig. 3 we show the spectrum of the observed mean intensity coming from a circle of radius $1^{\prime \prime}$ inside the detached shell. Remarkable red- and blue-shifted peaks can 


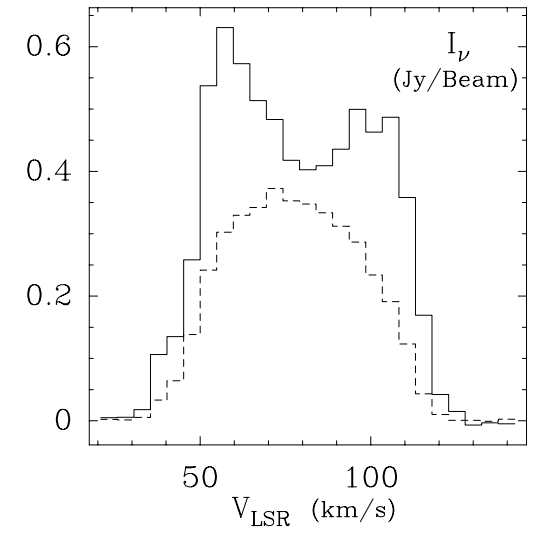

Fig. 3. The average intensity of the transition $\mathrm{SiO} v=0 \mathrm{~J}=$ $2-1$ in different areas of the maps (Fig. 1), as a function of the LSR velocity. The solid line represents the mean intensity inside a circle of radius $1^{\prime \prime}$, and the dashed line that inside a ring of radii $1^{\prime \prime}$ and $3^{\prime \prime}$, both centered in $(0,0)^{\prime \prime}$

be seen in this profile. The spectrum obtained for a concentric ring, of radii $1^{\prime \prime}$ and $3^{\prime \prime}$, peaks at the central velocity channels.

It is difficult to explain the high abundance of $\mathrm{SiO}$ in this very large and detached shell (see Sect. 1.2). $\mathrm{SiO}$ could be extracted from grains in shocked regions (that would also be particularly dense and hot). Indeed, we can speculate that this rapidly evolving star has shown successive phases of mass ejection with different velocities, which may lead to the development of shock fronts. In such a case we would be observing a structure that is so far unique: a quasi spherical shock propagating in an envelope around an evolved star. It is also possible that silicon is extracted by grain sputtering due to the large relative velocity between gas and dust particles expected for these sources, characterized by their high luminosity and expansion velocity (see Covatto \& Aannestad 2000 and references therein). In this case, we would be just detecting the inner layers of the dense envelope.
Anyhow, our knowledge on the extended shell responsible for the $\mathrm{SiO}$ emission is still poor. Our observations have shown unexpected properties of the circumstellar envelope of IRC +10420 , but we think that it is premature to attempt a comprehensive view of its structure and excitation conditions.

Acknowledgements. This work has been financially supported by the Spanish DGES, under project PB96-0104.

\section{References}

Bujarrabal, V., Gómez-González, J., \& Planesas, P. 1989, A\&A, 219, 256

Bujarrabal, V., \& Cernicharo, J. 1994, A\&A, 288, 551

Bujarrabal, V., Alcolea, J., \& Neri, R. 1998, ApJ, 504, 915

Covatto, C., \& Aannestad, P. A. 2000, MNRAS, 318, 67

de Jager, C. 1998, A\&AR, 8, 145

Goldreich, P., \& Scoville, N. 1976, ApJ, 205, 144

Hrivnak, B. J., Kwok, S., \& Volk, K. M. 1989, ApJ, 346, 265

Humphreys, R. M., Smith, N., Davidson, K., et al. 1997, AJ, 114,2778

Jones, T. J., Humphreys, R. M., Gehrz, R. D., et al. 1993, ApJ, 411,323

Kastner, J. H., \& Weintraub, D. A. 1995, ApJ, 452, 833

Klochkova, V. G., Chentsov, E. L., \& Panchuk, V. E. 1997, MNRAS, 292, 19

Loup, C., Forveille, T., Omont, A., \& Paul, J. F. 1993, A\&AS, 99,291

Lucas, R., Bujarrabal, V., Guilloteau, S., et al. 1992, A\&A, 262,491

MacKay, D. D. S., \& Charnley, S. B. 1999, MNRAS, 302, 793

Meixner, M., Ueta, T., Dayal, A., et al. 1999, ApJS, 122, 221

Nedoluha, G. E., \& Bowers, P. F. 1992, ApJ, 392, 249

Neri, R., Kahane, C., Lucas, R., Bujarrabal, V., \& Loup, C. 1998, A\&AS, 130, 1

Olofsson, H., Bergman, P., Eriksson, K., \& Gustafsson, B. 1996, A\&A, 311, 587

Ridgway, S. T., Joyce, R. R., Connors, D., Pipher, J. L., \& Dainty, J. C. 1986, ApJ, 302, 662

Willacy, K., \& Millar, T. J. 1997, A\&A, 324, 237 